\title{
Part-whole information assists in topological $\times$ topological but not in orientation $\times$ orientation conjunction searches
}

\author{
Weijia Chen • Anthea G. Blunden • Piers D. L. Howe
}

Published online: 23 January 2015

(C) The Psychonomic Society, Inc. 2015

\begin{abstract}
Visual search is a routine task used in everyday life and is an important field of research in cognitive psychology. In laboratory settings, it has been shown that search for a target defined by a unique conjunction of two colours is more efficient if one colour surrounds the other (a part-whole search) compared to when no such hierarchical structural relationship exists (a part-part search; Wolfe et al. in Perception \& Psychophysics, 55, 537, 1994). A similar result has been shown to hold for size $\times$ size conjunction searches (Bilsky \& Wolfe in Perception \& Psychophysics, 57, 749, 1995). We show that this result also holds for topology $x$ topology conjunction searches (where the stimuli are either hollow or filled), but not for orientation $\times$ orientation conjunction searches. We use the simultaneous-sequential paradigm to investigate a possible reason for the inefficiency of part-whole orientation search compared with the efficiency of part-whole searches of other features. We argue that two different attribute values from the same dimension can be processed independently, without interfering with each other for colour, size, and topology, but not for orientation. Because it is obviously more efficient to process a conjunction stimulus when both components of the conjunction can be processed without mutual interference, it follows that colour $\times$ colour, size $\times$ size, and topological $\times$ topology part-whole conjunction searches are likely to be more efficient than orientation $\times$ orientation partwhole conjunction searches.
\end{abstract}

Keywords Visual search - Attention: selective $\cdot$ Attention: interactions with memory

W. Chen · A. G. Blunden • P. D. L. Howe $(\bowtie)$

School of Psychological Sciences, University of Melbourne,

Melbourne, VIC, Australia

e-mail: pdhowe@unimelb.edu.au
In everyday and professional life, we often need to perform visual search tasks, such as looking for a friend at a train station, or spotting tumours on an x-ray. In a typical laboratory visual search task, an observer looks for a designated target item among a variable number of distractor items. Performance often is quantified as the average time taken to locate the target as a function of the number of distractor items (i.e., the set size; Treisman \& Gelade, 1980). The reciprocal of this measure is known as the search efficiency (Wolfe, 1998). Search efficiency reflects how well attention can be guided towards the target (Chun \& Wolfe, 1996; Wolfe, FriedmanHill, \& Bilsky, 1994). When a target differs from the distractors on a single attribute (e.g., colour, size, orientation, etc.), search is generally fast (Treisman \& Gelade, 1980; Wolfe, 2007; Wolfe, Cave, \& Franzel, 1989). Such searches often are termed feature searches as attention can be directed to the target based on its unique feature. Alternatively, the target and distractors may share the same attributes and only differ in the way that the attributes are combined. These searches are usually referred to as conjunction searches.

In some situations even conjunction searches can be quite efficient. Typically, this occurs when the attributes are derived from different dimensions, for example a colour $\times$ orientation conjunction search, such as searching for a red vertical target rectangle among distractors, half of which are green and vertical rectangles, and the remainder of which are red and horizontal rectangles (Treisman \& Sato, 1990; Wolfe et al., 1989; Wolfe et al., 1990; Wolfe, Yee, \& Friedman-Hill, 1992). Conversely, where attributes of a conjunctive search are derived from the same attribute dimension, search is generally inefficient (e.g. Wolfe et al., 1990). However, there is a notable exception to this rule. Within-dimension conjunction searches may be efficient if the attributes exhibit a structural hierarchy (Bilsky \& Wolfe, 1995; Wolfe et al., 1994). We use the term "hierarchy" in the same restricted 
sense as it was used in these previous studies: to refer to the case when the outer region of an object differs from its inner region.

Wolfe and colleagues (1994) found that observers had difficulty locating an object whose left side was red and whose right side was yellow among distractors, some of which had yellow right sides and blue left sides, whereas those remaining had red left sides and blue right sides (Fig. 1, top panel). Search was relatively inefficient, with the average search slope being $38.9 \mathrm{~ms} /$ item on target-present trials. However, observers were significantly more efficient in searching for a red object with yellow parts among blue objects with yellow parts and red objects with blue parts (Fig. 1, bottom panel). Here, the average search slope was just $13.1 \mathrm{~ms} /$ item on target-present trials. In the former case, the search was said to be for a part-part target as both parts of the target were a different colour. Conversely, in the latter case, the search was said to be for a part-whole target as the whole, i.e., the body of the object, was one colour while the inner parts were a different colour.

The hierarchical advantage for within-dimension conjunction search was replicated in a later study using size $\times$ size conjunctions (Bilsky \& Wolfe, 1995). In that study, the average part-whole search slope for target-present trials was $11.3 \mathrm{~ms} /$ item, significantly less than the average part-part target-present search slope of $28.7 \mathrm{~ms} /$ item.

However, from Bilsky and Wolfe (1995) it was unclear whether an equivalent result held for orientation $\times$ orientation conjunction searches. Bilsky and Wolfe created orientation $\times$ orientation conjunction stimuli using rectangles and bars. In a part-whole condition participants searched for a vertical rectangle (the "whole") with an embedded tilted bar (the "part")

\section{Part-Part Colour X Colour Search Target: Red \& yellow house} Distractors: Red \& blue house, blue \& yellow house
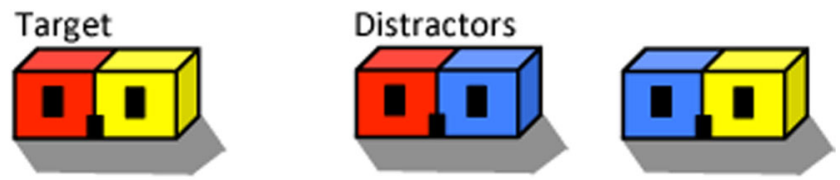

\section{Part-Whole Colour X Colour Search Target: Red house with yellow windows Distractors: Blue house with yellow windows, red house with blue windows}
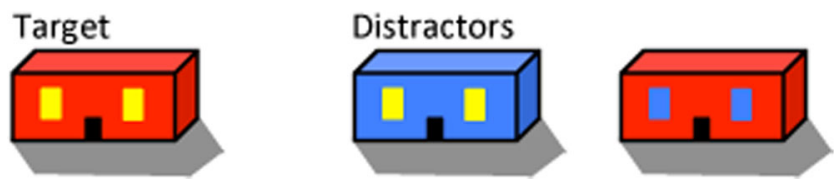

Fig. 1 Some of the stimuli used in Wolfe et al. (1994). Modified with permission from Wolfe et al. (1994, Perception, \& Psychophysics, 55, 537). among distractors of vertical rectangles with embedded horizontal bars and horizontal rectangles with embedded tilted bars. The authors reasoned that if structural information regarding orientation can guide attention, then participants should be able to efficiently search for a vertical rectangle with a diagonal part. However, average search slope for the part-whole stimuli was $54.5 \mathrm{~ms} /$ item on target-present trials, which was relatively inefficient compared to other withindimension, part-whole searches.

Bilsky and Wolfe (1995) suggested that part-whole orientation $\times$ orientation searches are inefficient because of the variability of orientation information in the natural world. They argued that colour and size are relatively invariant properties of objects, but orientation had a higher likelihood of variation. They gave the example of searching for a book. A yellow book with black print would still look like a yellow book with black print even if it were moved; the same applies to a large book with small print. But a vertical book with horizontal print on it could easily be rotated and become a horizontal book with vertically oriented print. Bilsky and Wolfe argued that the visual system only invested in the parallel processing of hierarchical information for the more invariant cues, so declined to invest in orientation.

While this work demonstrated that part-whole orientation $\times$ orientation searches are relatively inefficient compared with other within-dimension part-whole searches, it did not address the question of whether structural hierarchy assists in orientation $\times$ orientation conjunction searches at all. To investigate this question, one would need to compare part-whole orientation $\times$ orientation search to the equivalent part-part orientation $\times$ orientation search. Bilsky and Wolfe (1995) did not run a part-part orientation $\times$ orientation search condition and could not perform this comparison. While previous work has investigated the efficiency of part-part orientation $\times$ orientation search (Wolfe et al., 1990), those stimuli were quite different from those used by Bilsky and Wolfe, thereby preventing a meaningful comparison from being made.

In addition to both colour and size, there are other features that are likely to display a hierarchical advantage for withindimension conjunction searches. For example, shape is another attribute found to efficiently guide attention in visual search (Wolfe \& Horowitz, 2004). Unlike an object's orientation, its shape is invariant under translations and rotations. It therefore follows from the above reasoning that there should be a hierarchical advantage for shape $\times$ shape conjunctive searches. There are many aspects to the concept of shape (Chen, 2005; Donnelly, Humphreys, \& Riddoch, 1991; Treisman \& Gormican, 1988). However, as topological status (e.g., solid vs. hollow) has shown to be particularly efficient at guiding attention (Chen, 2005), our study concentrated on topology.

To foreshadow our results, we found that like colour $\times$ colour, and size $\times$ size conjunction searches, topology $\times$ 
topology conjunction searches were efficient when a hierarchical structure was present (i.e., part-whole topology $\times$ topology searches were more efficient than the corresponding part-part topology $\times$ topology searches). However, extending Bilsky and Wolfe (1995), we found no hierarchical benefit for orientation.

A potential reason for this finding comes from the visual short-term memory (VSTM) literature (Becker, Miller, \& Liu, 2013; Mance, Becker, \& Liu, 2012). Mance and colleagues (2012) presented participants with coloured squares either simultaneously or sequentially, with participants performing either a recognition task, responding "yes" or "no" as to whether a probe colour had previously been shown, or a recall task, where participants named the colour which had appeared at a cued location. Because they found that their participants were just as accurate in the simultaneous condition as in the sequential condition, they concluded that two colours can be processed simultaneously without mutual interference.

Becker and colleagues (2013) replicated Mance et al.'s (2012) study using differently oriented gratings. Contrary to the findings for colour, they found an advantage for sequential processing over simultaneous processing. This suggests that two different orientations cannot be processed simultaneously without mutual interference. This could potentially explain why orientation $\times$ orientation conjunction searches are always inefficient. Attempting to simultaneously process two orientations results in mutual interference, rendering the search inefficient, regardless of any part-whole structure. We extended this paradigm into the topology and size domains. We found that the size or topology of two objects could be processed simultaneously without mutual interference. This result is consistent with the findings for colour (Mance et al., 2012) but contradict those for orientation (Becker et al., 2013). As such, they provide a potential explanation why a hierarchical benefit is observed for colour, topology and size but not for orientation.

\section{Experiment 1: Topology $\times$ Topology Conjunction Search}

In Experiment 1, we used solid and hollow circles to examine topology $\times$ topology conjunction searches. If shape information is processed similarly to colour and size, then part-whole topology $\times$ topology conjunction search should be more efficient than part-part topology $\times$ topology conjunction search.

Method

Participants Thirteen participants, aged 18 to 55 years, took part in the experiment. We chose this number of participants because Bilsky and Wolfe (1995) had shown robust effects using similar stimuli with a similar number of participants. All participants had corrected or uncorrected acuity of at least 20/

25 (near-field Snellen eye chart) and normal colour vision (Ishihara plates). All participants gave informed consent and were compensated with $\$ 15$ for their participation.

Materials Figure 2 shows the stimuli for Experiment 1. Stimuli were large and small circles. To make the parts and wholes easily distinguishable, all large circles were coloured green $\left(\right.$ CIE coordinates $\mathrm{x}=0.23, \mathrm{y}=0.68$, luminance $=70 \mathrm{~cd} / \mathrm{m}^{2}$ ) and all small circles were coloured red (CIE coordinates $\mathrm{x}=$ $0.64, \mathrm{y}=0.33$, luminance $=24 \mathrm{~cd} / \mathrm{m}^{2}$ ). At the $60-\mathrm{cm}$ viewing distance, the large circle had a radius of $1.96^{\circ}$ and the small circle had a radius of $0.67^{\circ}$. In the part-whole topology $\times$ topology conjunction condition, participants searched for a solid outer circle with a hollow inner circle. In the part-part topology $\times$ topology conjunction condition, participants searched for the item with both a hollow circle and a solid circle inside. In the part-feature condition, participants searched for a small hollow circle among small solid circles. In the whole-feature condition, the target was a large solid circle and the distractors were large hollow circles. These stimuli were presented on a black background (luminance $\left(<0.01 \mathrm{~cd} / \mathrm{m}^{2}\right)$, except for the part-feature stimuli which were presented on a green background (CIE coordinates and luminance of this background was the same as the green "wholes"), as the parts in the other conditions were also presented on a green background. The backgrounds could be seen through the unfilled portions of those stimuli that were hollow.

Procedure The stimuli were presented on a personal computer using MATLAB ${ }^{\circledR}$ and the Psychophysics Toolbox (Brainard, 1997; Pelli, 1997). The items were presented in 4, 10 , or 16 pseudorandom locations in a $4 \times 4$ array of possible

Part-whole:

Target

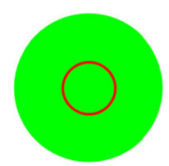

Part-part:
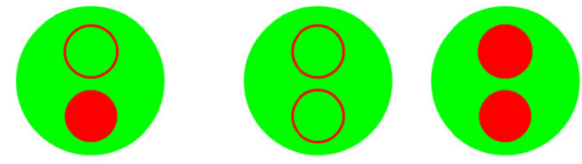

Part-feature:

Whole-feature:
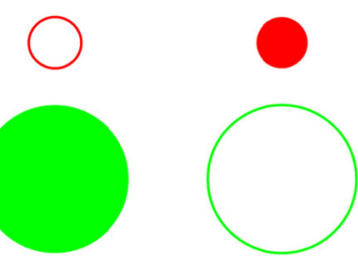

Fig. 2 Stimuli for Experiment 1: topology $\times$ topology conjunctions. All stimuli were presented on a black background. In the cases of the hollow stimuli, this background could be seen through the unfilled portions of the stimuli. 
locations. The stimulus array subtended an area of $26.7^{\circ} \times 34.9^{\circ}$ at the $60-\mathrm{cm}$ viewing distance. Other than the obvious constraint that no two stimuli could be assigned to the same location, there were no constraints on the location assignments. To prevent the items being aligned, each item in the array was offset by a random amount chosen from a uniform distribution in the range $-0.03^{\circ}$ to $+0.03^{\circ}$ along both the horizontal and vertical dimensions. A target item was present in the display on one half of the trials. Participants responded with a key press to indicate target presence or absence. Response accuracy and response time (RT) were recorded by the computer. The maximum presentation time was $3,000 \mathrm{~ms}$. If a participant failed to respond within this time, RT was not recorded and that trial was discarded and redone. Participants were given 10 practice trials and were tested on 120 trials in each condition, for a total of 490 trials. An equal number of trials were presented for each set size. Within the constraints previously mentioned, set size and target presence were pseudorandom across trials within a condition. Order of conditions was pseudorandom across participants.

\section{Results}

For each participant and each condition mean RTs were calculated for each set size for target-present (present trials) and target-absent (blank) trials. Slopes and y-intercepts of the RT $\times$ Set Size functions were computed by linear regression. Figure 3 shows average RT as a function of set size for all four conditions. Average search slopes (standard error of the mean, SEM, in brackets) for the part-whole condition was 5.0 (2.8) $\mathrm{ms} /$ item for target-present trials and 27.8 (4.7) $\mathrm{ms} /$ item for blank (target-absent) trials. Average search slope for the part-part condition was 46.8 (4.7) ms/item for target-present trials and 95.3 (6.2) for blank trials. Average search slope for the part-feature condition was $5.2(2.3) \mathrm{ms} /$ item for targetpresent and $8.6(2.8) \mathrm{ms} /$ item for target-absent. Average search slope for whole-feature condition was $0.7(1.0) \mathrm{ms} /$ item for the target-present trials and $-1.5(1.6) \mathrm{ms} /$ item for the blank trials. Mauchly's test of sphericity indicated that the assumption of sphericity had not been violated, $\mathrm{W}(5)=0.372, p=0.061$. A within-subjects ANOVA revealed a significant difference among the four experiment conditions, $\mathrm{F}(3,36)=53.2, p<$ 0.001 , partial $\eta^{2}=0.816$. A $t$-test was performed to compare search efficiencies between the part-whole and part-part conditions. The search slope for the part-whole topology $\times$ topology conjunction search was significantly smaller than the search slope for the part-part topology $\times$ topology conjunction search, $t(12)=8.17, p<0.001$, Cohen's $d=2.36$. Both partand whole-feature searches were highly efficient, so it is unlikely that the steeper search slope in the part-part condition was due to difficulty in searching for the constituent features. A part-whole benefit was therefore observed for topology.

The advantage for the part-whole condition cannot be attributed to a speed-accuracy trade off. The error rate in the part-whole condition was in fact slightly less than that in the part-part condition thereby demonstrating that observers were not being more careful and thus slower in the part-part condition (see accuracy data, Appendix).

\section{Discussion}

In Experiment 1, we found evidence supporting a part-whole advantage in the processing of topological information. One possible alternative explanation for this finding could be that the search was based on colour and not topology. For

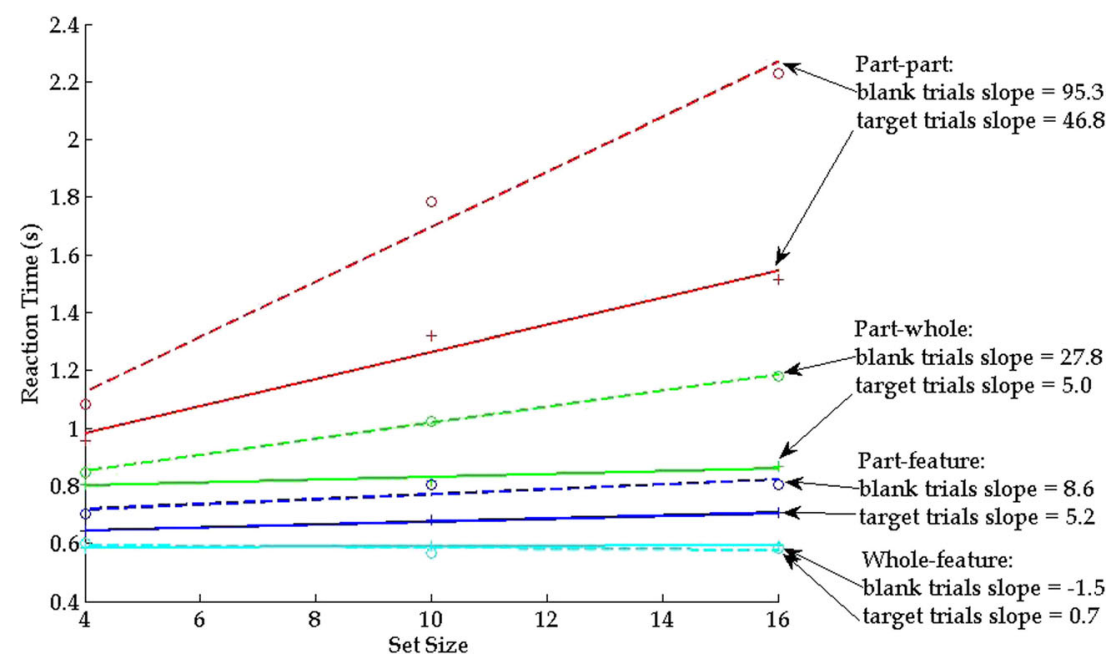

Fig. 3 Results for Experiment 1: topology $\times$ topology conjunction search yielded steep search slopes for part-part stimuli and shallower slopes for part-whole stimuli. In this and subsequent graphs, target-present trials are plotted with solid lines, and target-absent (i.e., blank) trials are potted with

dashed lines. Part-whole searches are plotted in green and part-part searches are plotted in red. Search slopes are lines of best fit obtained via linear regression. 
example, in the part-whole condition, the target item consistently had the largest amount of green, and therefore search could have taken place based on this feature alone. However, this strategy would not have worked for the part-part condition, because the target was no longer the object with the most amount of green. This could potentially explain why search for the target in the part-part condition was less efficient than the search for the target in the part-whole condition. This potential confound was addressed in Experiment 2.

\section{Experiment 2: Topological search with additional distractors}

To address this potential confound we introduced a new distractor type: objects that were entirely green. Thus, the target would never be the object with the most amount of green so could not be searched for on the basis of colour alone.

\section{Method}

Participants Thirteen participants, aged 18 to 30 years, were tested. As before, all participants had corrected or uncorrected acuity of at least 20/25 (near-field Snellen eye chart) and normal colour vision (Ishihara plates). All participants gave informed consent and were rewarded with $\$ 15$ for their participation.

Materials The stimuli were identical to those used in Experiment 1 except that a large green disc (radius, $1.96^{\circ}$ ) was introduced as a third distractor type in the part-whole and part-part conditions. This ensured that the target in these conditions was no longer the item with the largest amount of green. Again, wholes were coloured green and parts were coloured red to make them easily distinguishable. The CIE coordinates, luminance, and methods were otherwise identical to those in the previous experiment. The new stimulus set is illustrated in Fig. 4.

\section{Results}

Figure 5 shows the average RT as a function of set size. Average search slope for the part-whole condition was 14.0 (3.4) $\mathrm{ms} /$ item for target-present and 44.7 (6.9) $\mathrm{ms} /$ item for target-absent trials. Average search slope in the part-part condition was 29.7 (3.5) ms/item for target-present trials and 56.6 (6.5) $\mathrm{ms} /$ item for blank trials. Average search slope for the part-feature was $1.1(1.2) \mathrm{ms} /$ item when the target was present and $16.0(2.2) \mathrm{ms} /$ item when the target was absent. Average search slope for the whole-feature condition was -2.4 (1.1) $\mathrm{ms} /$ item for target-present trials and $-1.9(2.6) \mathrm{ms} /$ item for

blank trials. Both feature searches were highly efficient. Mauchly's test of sphericity indicated that the assumption of sphericity had been violated, $\mathrm{W}(5)=0.313, p=0.03$, so we used the Geenhouse-Geisser correction. A within-subjects ANOVA showed a significant difference among the conditions, $F(2.06,24.7)=34.8, p<0.001$, partial $\eta^{2}=0.744$. A repeated-measures $t$-test was conducted to compare the magnitude of search slopes in the part-whole and part-part conditions. The part-whole topology $\times$ topology conjunction search had a significantly smaller search slope than the part-part topology $\times$ topology conjunction search, $t(12)=3.38, p=$ 0.005 , Cohen's $d=0.935$. The error rate was almost identical in the part-whole condition and in the part-part condition indicating that this data cannot be accounted for by a speedaccuracy trade off (Appendix). These results therefore confirm that a part-whole advantage exists for topology $\times$ topology conjunctions.

\section{Discussion}

Experiment 2 addressed the concern that search in the part-whole condition may be guided solely on the basis of colour. Neither target in the part-whole or part-part condition contained the most amount of green or red; therefore, attention could not be guided to the targets based solely on colour. Of course, it could still be argued that colour might help guide attention. For example, if observers adopted a strategy whereby they searched for the item with the most amount of green in the part-whole condition, they would likely find the target in the first third of the items that they attended to, thereby potentially reducing the number of items that they would need to inspect. A similar strategy could be applied to the partpart condition. Searching for the item with a large amount of red also would mean that the target would likely be found in the first third of the items inspected. Thus, if

Part-whole:

Target

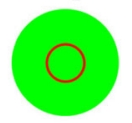

Part-part:
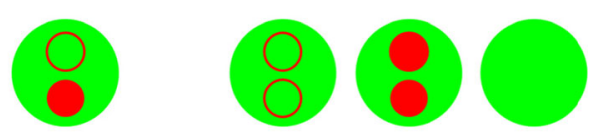

Part-feature:

Whole-feature:
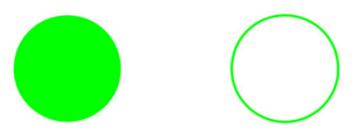

Fig. 4 Stimuli for Experiment 2: topology $\times$ topology conjunction search with an additional distractor type. 


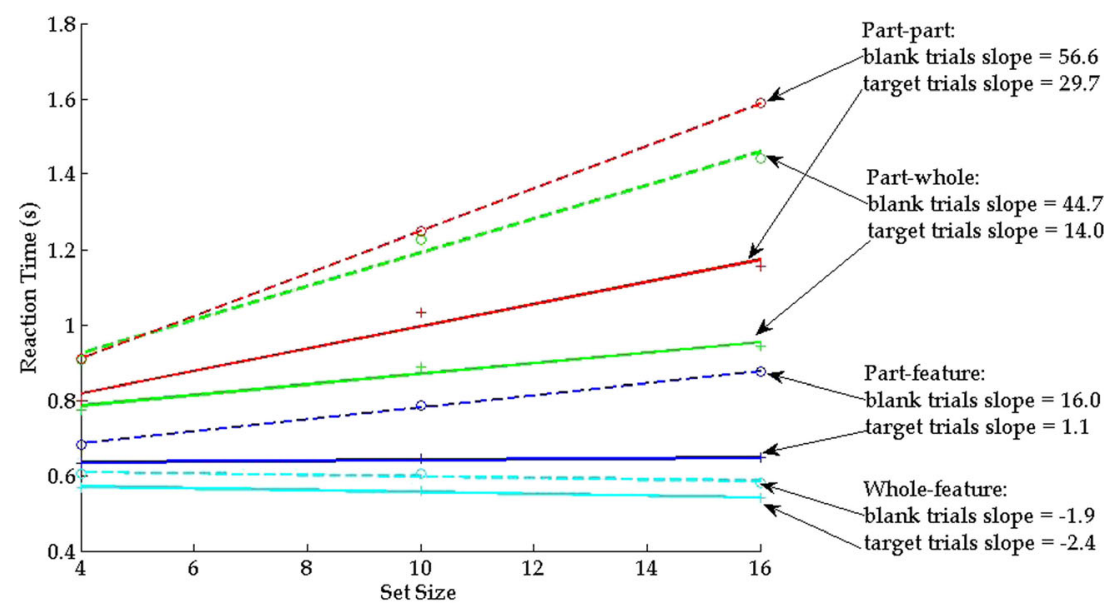

Fig. 5 Results for Experiment 2: topology $\times$ topology conjunction search again yield steeper search slopes for part-part stimuli and shallower slopes for part-whole stimuli.

colour can help guide attention, we would expect that the search slopes for the part-whole and part-part conditions to be the same. Because this is not the case, we believe that, in this particular experiment, colour on its own did not play a major role in guiding attention.

\section{Experiment 3: Aspect Ratio $\times$ Aspect Ratio Conjunction Search}

To further investigate the degree to which shape $\times$ shape conjunction searches could benefit from structural hierarchy, another aspect of shape was tested by varying the aspect ratio of parts and wholes. Experiment 3 used rectangles and squares to create aspect ratio $\times$ aspect ratio conjunction search. Following the findings in Experiment 1 and 2, it was expected that part-whole aspect ratio $\times$ aspect ratio conjunction search should be more efficient than part-part aspect ratio $\times$ aspect ratio conjunction search, hence yield a shallower search slope.

\section{Method}

Participants Twelve participants, aged 18 to 55 years, were tested. As before, all had corrected or uncorrected acuity of at least 20/25 (Snellen eye chart) and normal colour vision (Ishihara plates). All participants gave informed consent and were rewarded with course credit for their participation.

Materials The stimuli are illustrated in Fig. 6. In the partwhole condition, participants searched for the item with an outer rectangle (whole) and an inner square (part). In the partpart condition, participants searched for the conjunction of an inner rectangle with an inner square among distractors with two inner rectangles or two inner squares. In the part-feature condition, participants searched for a small square among small rectangles. In the whole-feature condition, the target item was a large rectangle and the distractors were large squares. All wholes were green, and all parts were red to make the wholes and parts easily distinguishable. The CIE coordinates and luminance were identical to those in the previous experiments. The size of the large rectangle was $4.87^{\circ} \times 1.24^{\circ}$, the size of the large square was $2.48^{\circ} \times 2.48^{\circ}$, the size of the small rectangle was $1.63^{\circ} \times 0.38^{\circ}$, and the size of the small square was $0.76^{\circ} \times 0.76^{\circ}$. The methods were otherwise similar to those in the previous experiment.

Results

The results of Experiment 3 are shown in Fig. 7. Average search slope for the part-whole condition was 65.4 (6.7) ms/ item for target-present and $136.2(9.6) \mathrm{ms} /$ item for targetabsent trials. Average search slope in the part-part condition was 82.6 (7.2) $\mathrm{ms} /$ item for target-present trials and 155.7 (9.4) $\mathrm{ms} /$ item for blank trials. Average search slope for the partfeature was $8.8(0.8) \mathrm{ms} /$ item when the target was present and

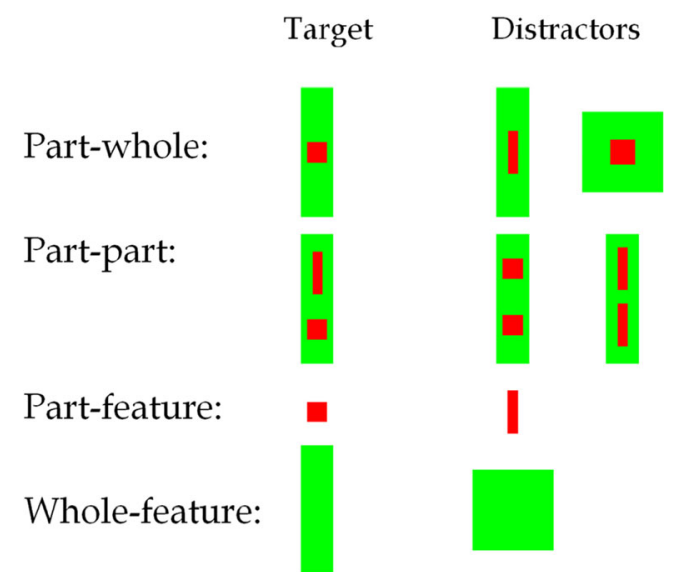

Fig. 6 Stimuli used to test if a search for a target defined by a conjunction of two aspect ratios benefits from part-whole hierarchical structure. 


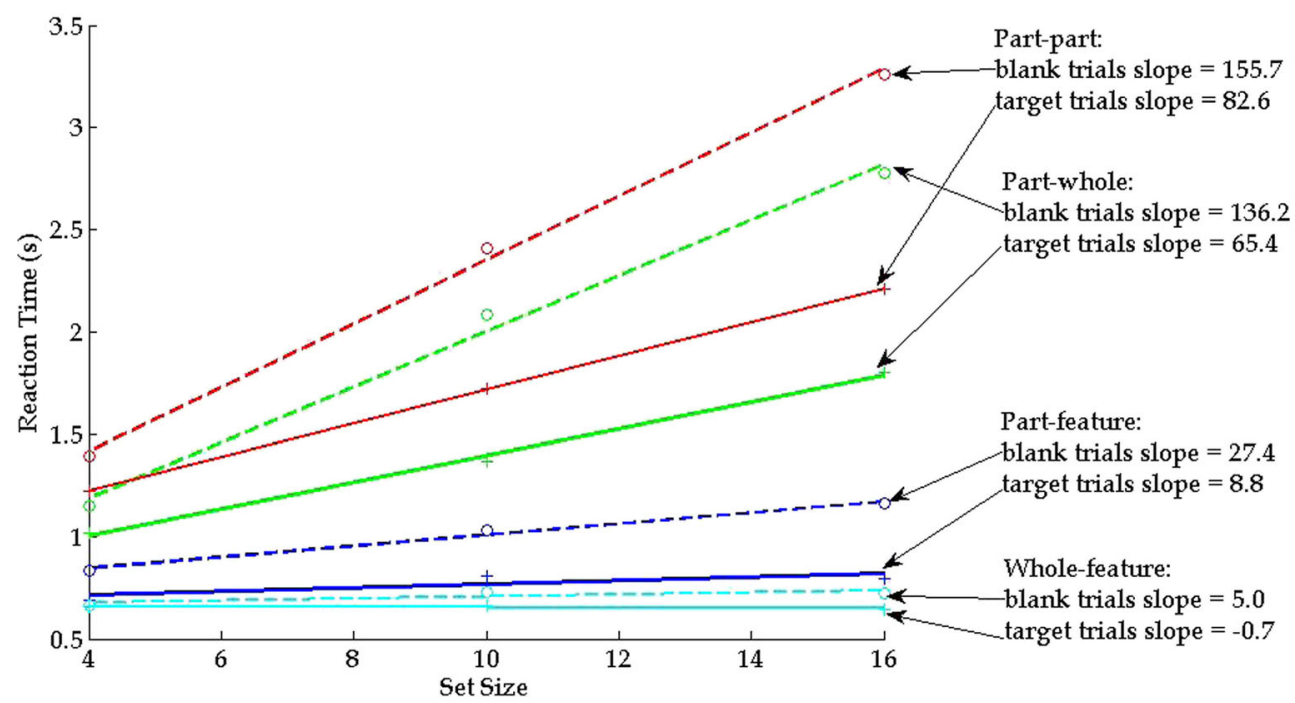

Fig. 7 Results for Experiment 3: aspect ratio $\times$ aspect ratio conjunctions again yield steeper search slopes for part-part stimuli and shallower slopes for part-whole stimuli.

27.4 (3.9) $\mathrm{ms} /$ item when the target was absent. Average search slope for the whole-feature condition was -0.7 (1.4) $\mathrm{ms} /$ item for target-present trials and $5.0(1.5) \mathrm{ms} /$ item for blank trials. The whole-feature search was highly efficient, and the partfeature search was reasonably efficient. Mauchly's test of sphericity indicated that the assumption of sphericity had been violated, $\mathrm{W}(5)=1.32, p=0.002$, so we applied the Greenhouse-Geisser correction when performing the ANOVA. A within-subjects ANOVA showed a significant difference among the conditions, $F(1.99,21.9)=72.7, p<$ 0.001 , partial $\eta^{2}=0.951$. A repeated-measures $t$-test revealed that the search slopes for the part-whole and part-part conditions were not quite significantly different, $t(11)=1.93, p=$ 0.08 .

\section{Discussion}

The results of this experiment are inconclusive. The search slopes for the part-whole and part-part conditions were almost significantly different $(p=0.08)$, suggesting that there might be a small part-whole advantage. However, the fact that the part-whole search was highly inefficient $(65.4 \mathrm{~ms} /$ item $)$ compared with part-whole searches involving topological status $(14.0 \mathrm{~ms} /$ item as tested in Experiment 2) argues against the notion that there is a part-whole advantage. Given these two findings, it is unclear whether or not a hierarchical advantage exists for aspect ratio. It could be that a hierarchical advantage does exist, but the advantage was too small to be detected by this particular experiment. Because we were unable to create a version of this experiment that gave a conclusive answer, we decided to move on to studying orientation $\times$ orientation conjunction searches.

\section{Experiment 4: Orientation $\times$ Orientation Conjunction Search}

From previous research, it is unclear whether a part-whole advantage exists for orientation $\times$ orientation conjunction searches. While it has been shown that part-whole orientation $x$ orientation searches are inefficient, it is unclear whether there is any hierarchical benefit at all for such searches because the part-whole orientation $\times$ orientation searches have never been compared with the equivalent part-part orientation $\times$ orientation searches (Bilsky \& Wolfe, 1995). Experiment 4 addresses this gap in the literature using stimuli similar to those employed in Bilsky and Wolfe (1995) except that we also included a part-part orientation $\times$ orientation conjunction condition to act as a point of comparison.

Method

Participants Thirteen psychology students, aged 18 to 24 years, from the University of Melbourne participated in the experiment. All had corrected or uncorrected acuity of at least 20/25 (near-field Snellen eye chart) and normal colour vision (Ishihara plates). All participants gave informed consent and were rewarded with course credit for their participation.

Materials Figure 8 shows the stimuli for Experiment 4 . Stimuli were vertically or horizontally oriented rectangles 
Target

Distractors

Part-whole:
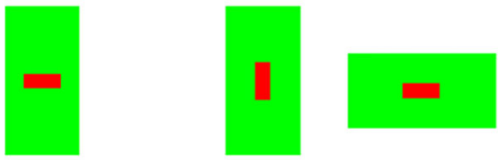

Part-part:
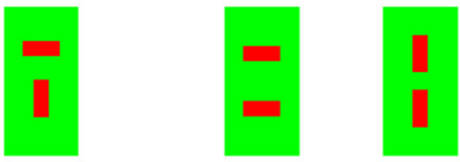

Part-feature:

Whole-feature:
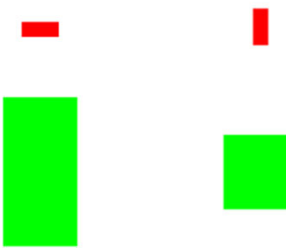

Fig. 8 Stimuli used in Experiment 3: orientation $\times$ orientation conjunction search.

with vertically or horizontally oriented rectangles inside. As before, to make the parts and wholes easily distinguishable, all outer rectangles were coloured green and all inner rectangles were coloured red. The CIE coordinates and luminance were identical to those in the previous experiment. At the $60-\mathrm{cm}$ viewing distance, the size of the outer rectangle was $1.91^{\circ} \times 0.96^{\circ}$ and the size of each inner rectangle was $0.19^{\circ} \times 0.48^{\circ}$. In the part-whole condition, participants searched for a vertical rectangle with a horizontal rectangle inside. In the part-part condition, participants searched for the item with both a horizontal rectangle and a vertical rectangle inside. In the partfeature condition, participants searched for a small, horizontal rectangle among small, vertical rectangles. In the whole-feature condition, the target was a large, vertical rectangle, and the distractors were large, horizontal rectangles. The methods were otherwise similar to those in the previous experiment.

Results

Figure 9 shows average RT as a function of set size for all four conditions. Average search slope for the part-whole condition was $88.2(8.7) \mathrm{ms} /$ item on target-present trials and $196.2(12.1) \mathrm{ms} /$ item on blank trials. Average search slope for the part-part condition was $104.6(8.7) \mathrm{ms} /$ item for target-present trials and $198.0(11.5) \mathrm{ms} /$ item for blank trials. Average search slope for the part-feature condition was $-0.7(1.7) \mathrm{ms} /$ item when the target was present and $40.1(6.3) \mathrm{ms} /$ item when the target was absent. Average search slope for the whole-feature condition was 4.5 (1.6) $\mathrm{ms} /$ item on target-present trials and 9.8 (2.9) ms/item on blank trials. Mauchly's test indicated that the assumption of sphericity had been violated, $\mathrm{W}(5)=0.150, p=0.001$, so we applied the Greenhouse-Geisser correction. A within-subjects ANOVA showed a significant difference among the four experimental conditions, $F(2.01,24.1)=$ 77.5, $p<0.001$, partial $\eta^{2}=0.866$. A repeated measures $t$-test was performed to compare search efficiencies between the part-whole and part-part conditions. The search slope for the part-whole orientation $\times$ orientation conjunction search was not significantly smaller than the search slope for the part-part orientation $\times$ orientation conjunction search, $t(12)=1.39, p=0.191$.

The mean error rates were comparable to those reported in Bilsky and Wolfe (1995), see Appendix. The mean error rate in the part-whole condition was slightly greater than that in the part-part condition ( $16.8 \%$ vs. $8.21 \%)$. This difference was statistically significant, repeated measures $t(12)=6.76, p<$ 0.001 , Cohen's $d=1.96$. This suggests that part-whole

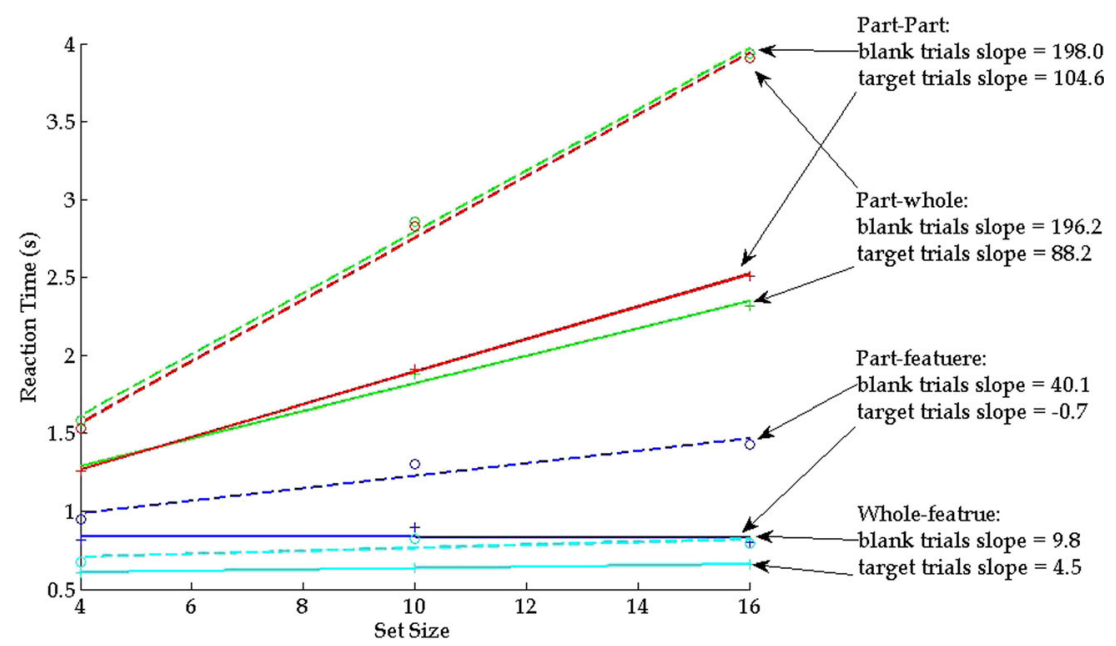

Fig. 9 Results for Experiment 3: orientation $\times$ orientation conjunctions yield very similar search slopes for part-part stimuli and part-whole stimuli. 
orientation $\times$ orientation searches may have actually been harder for the observers to perform than part-part orientation $\times$ orientation searches.

\section{Discussion}

So far, we have found that hierarchical information assists in topology $\times$ topology but not orientation $\times$ orientation conjunction searches. Previous studies have found evidence that hierarchical information assists in colour $\times$ colour and size $\times$ size conjunction searches (Wolfe et al, 1994; Bilsky \& Wolfe, 1995). Taken together, these findings suggest that the perceptual processing of orientation could be fundamentally different from the processing of colour, size, and topology. As discussed in the introduction, Bilsky and Wolfe (1995) attempted to explain why hierarchical information does not benefit orientation $\times$ orientation conjunction searches in terms of attribute variability in the natural world. They argued that orientation information is more variable than colour or size information and that the visual system only invests in the parallel processing of hierarchical information for the more invariant cues (i.e., not orientation).

Our main concern with this claim is that Bilsky and Wolfe (1995) did not specify how the variability of a dimension should be measured or how invariant a dimension needs to be for searches to benefit from hierarchical information. Consequently, it is difficult to use their theory to make meaningful predictions. For example, most large objects (and some small objects) actually do have a standard orientation. For example, buildings, lamp posts, trees, houses, and furniture would mostly appear in the upright orientation. It is therefore not clear why their theory would necessarily predict that partwhole orientation $\times$ orientation searches should be inefficient. For this reason, we favour an alternative explanation of our results. To recap, studies of VSTM capacity using the simultaneous-sequential paradigm found that two colours could be registered without mutual interference, but two orientations could not (Becker, et al., 2013; Mance, et al., 2012). It follows that if two orientations cannot be registered without mutually interfering with each other, then this reduces the efficiency with which the two components of a part-whole orientation $\times$ orientation search can be processed, which in turn would degrade the efficiency of such searches. This could explain why part-whole orientation $\times$ orientation conjunction searches are inefficient.

This theory makes some clear predictions. Where there is a hierarchical benefit for a within-dimension conjunction search, it follows that it should be possible to register two examples of that dimension without mutual interference. Colour $\times$ colour, size $\times$ size, and topology $\times$ topology conjunction searches have all been shown to benefit from part-whole processing. Furthermore, it has already been shown that observers can register two colours simultaneously without mutual interference (Mance, et al., 2012). We predict that observers should similarly be able to register two topological features or two sizes simultaneously without mutual interference. In Experiments 5 and 6, we test this prediction using the simultaneous-sequential paradigm.

\section{Experiment 5 \& 6: Simultaneous-Sequential Paradigm}

In Experiment 5, we tested whether observers could register two topological features simultaneously without mutual interference. Specifically, we tested whether recall was worse when two topological features were presented together (the simultaneous condition), compared to when they were presented one after the other (the sequential condition). According to the logic of the simultaneous-sequential paradigm, if two features can be processed without mutual interference then there should be no difference in recognition accuracy if the two features are presented simultaneously or sequentially. Conversely, if the two features can only be processed one at a time, or at least if the processing of one feature interferes with the processing of a second feature, then recognition performance should be greater when the features are presented sequentially compared to when they are presented simultaneously. In Experiment 6, we used the same simultaneoussequential paradigm to investigate whether the size of two objects could be registered without mutual interference. For the two experiments, we expect the recall accuracy for two topological features (Experiment 5) or two sizes (Experiment 6) to be the same regardless of whether the two topological features or the two sizes were presented simultaneously or sequentially.

\section{Method}

Participants Thirteen observers, aged 20 to 27 years, participated in both experiments, with experiment order randomly assigned for each participant. Data from two participants were excluded due to relatively poor vision (20/32). The remaining participants all had corrected or uncorrected acuity of at least $20 / 25$, as measured by a near-field Snellen eye chart. All participants gave informed consent and were compensated at the rate of $\$ 12 / \mathrm{h}$.

Materials As before, the stimuli were presented on a personal computer using MATLAB ${ }^{\circledR}$ and the Psychophysics Toolbox (Brainard, 1997; Pelli, 1997). In Experiment 5, the stimuli were solid or hollow circular white discs with a diameter of $1.1^{\circ}$ and a luminance of $99.0 \mathrm{~cd} / \mathrm{m}^{2}$. In Experiment 6 , the discs were solid and white but were either large $\left(1.1^{\circ}\right)$ or small $\left(0.55^{\circ}\right)$. All stimuli were presented on a black background $\left(<0.01 \mathrm{~cd} / \mathrm{m}^{2}\right)$. The viewing distance was $60 \mathrm{~cm}$. A fixation 
cross $\left(0.2^{\circ}\right)$ was presented in the centre of the screen. The discs could be presented in one of four possible locations, located at the corners of an imaginary square (eccentricity, $2.1^{\circ}$ ) centred on fixation. The mask stimulus was a square made up of $8 \times 8$ pixels (total size, $1.1^{\circ}$ ). The luminance of each pixel was randomly selected from the range $<0.01 \mathrm{~cd} / \mathrm{m}^{2}$ to $99.0 \mathrm{~cd} / \mathrm{m}^{2}$.

Procedure The procedure was the same in both experiments and was similar to that employed by Becker et al. (2013). In the sequential condition, two test discs were presented one after the other in two locations (out of the four possible locations, randomly determined on each trial). The presentation of each test disc was followed by a mask that lasted $42 \mathrm{~ms}$. A 496-ms retention interval separated the first mask and the second test stimulus, and a second 496-ms retention interval period followed the presentation of the second mask. A square outline (size, $1.1^{\circ}$; line thickness, $0.1^{\circ}$ ) then appeared as a probe at one of the disc locations and remained on screen until a response was made. Two words were presented on the screen ("hollow" and "solid" in Exp. 5; "large" and "small" in Exp. 6), and participants were asked to click on the word that corresponded to the probed location. The simultaneous condition was identical to the sequential condition, except that two discs were presented at the same time, followed by two simultaneous masks, and a single 496-ms retention period. The procedure for Experiment 5 is illustrated in Fig. 10.

Both the simultaneous and sequential conditions were run in blocks of 60 trials. A prompt appeared at the start of each block informing participants of the block type (i.e., simultaneous or sequential). There were four blocks in total, two in each condition. The order of blocks was randomised for each participant.

Calibration procedure Before participating in the main task, all of the participants performed five intermixed simultaneous and sequential practice trials. In these trials, stimuli were presented for a fixed duration of $248 \mathrm{~ms}$. This was followed by a calibration task where observers performed two blocks of simultaneous and two blocks of sequential trials. The order of these blocks was randomised. During this task, the stimulus exposure time varied according to the method of constant stimuli. Eight possible equally spaced durations were used between $11.8 \mathrm{~ms}$ and $94.1 \mathrm{~ms}$, corresponding to between 1 to 8 video frames at the $85-\mathrm{Hz}$ monitor refresh rate. Each block consisted of 64 trials, eight at each stimulus duration. We used only the data from the two sequential blocks to find individual participant thresholds. The simultaneous blocks were included to ensure that participants had equivalent practice with both conditions.

We calculated the observer's percentage correct at each stimulus duration for the sequential trials. Following the method of Becker et al. (2013), this accuracy data was fitted with an exponential psychometric function:

$p c=\delta+\gamma\left(1-e^{\beta t}\right)$

where $\mathrm{pc}$ is the percentage correct, $\mathrm{t}$ is the exposure duration, and $\delta, \gamma$, and $\beta$ are free parameters. The data were fitted using the MATLAB lsqcurvefit function and the stimulus
Fig. 10 Schematic of the two conditions in Experiments 5 and 6 . In the sequential condition (left), two discs appeared, one at a time. In the simultaneous condition (right), two discs appeared at the same time. The duration that each disc was presented was based on the individual observer's threshold and was held constant across conditions. A noise mask followed each disc. A location probe (square outline) indicated the disc for the response at the end of each trial. Participants were asked to identify whether the probed disc was filled or hollow (Exp. 5) or large or small (Exp. 6).

\section{Sequential Condition \\ Simultaneous Condition}

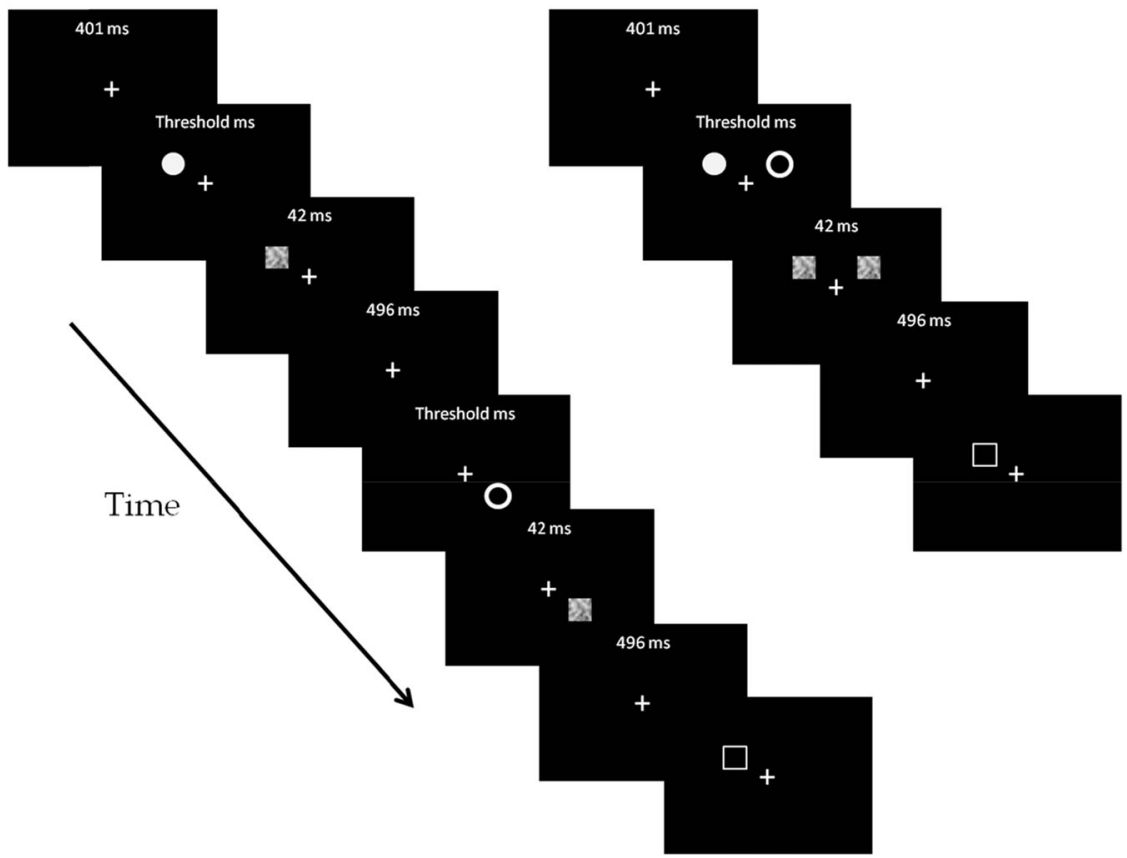


duration that produced $75 \%$ correct was used for all conditions in the main task.

\section{Results}

The mean stimulus duration for Experiment 5 was $21.8 \mathrm{~ms}$ (duration range 11.8-35.4 ms, $S D=9.4 \mathrm{~ms}$ ) and for Experiment 6 was $52.7 \mathrm{~ms}$ (duration range 23.6-105.9 ms, $S D=$ $27.7 \mathrm{~ms}$ ). The accuracy for each of the two conditions in Exp. 5 (topology) and Exp. 6 (size) were calculated for each participant. The average percentage correct for both conditions in both experiments is shown in Fig. 11. A paired $t$-test revealed no significant difference in accuracy between the simultaneous and sequential conditions for topology [Experiment 5: $t(10)=1.82, p=.10$ ] or for size [Experiment $6: t(10)=0.03, p$ $=.98]$. Indeed, in both cases performance was slightly greater in the simultaneous condition, the opposite of what would be predicted if there was mutual interference.

\section{Discussion}

The fact that there was no significant difference in accuracy between the simultaneous and sequential conditions for both topology and size suggests that for these dimensions two features can be simultaneously processed without mutual interference. A similar result has been shown for colour but not for orientation (Becker, et al., 2013; Mance, et al., 2012). Taken together, these results suggest that the hierarchical advantage seen in colour, size, and topology may be due to the fact that for these dimensions two features can be simultaneous processed without mutual interference. Conversely,

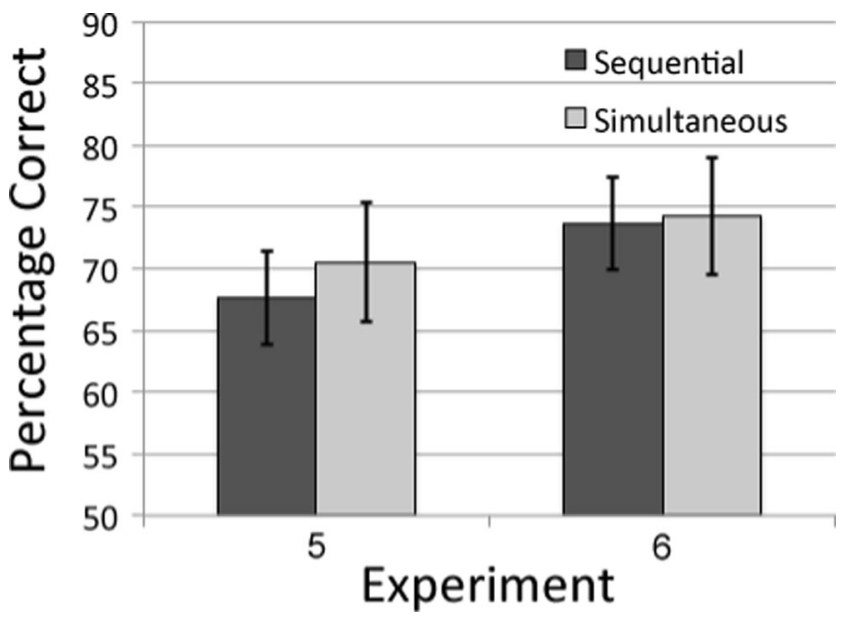

Fig. 11 Results for Experiment 5 (topology) and Experiment 6 (size). Error bars represent within-subject $95 \%$ confidence intervals (Cousineau, 2005; Morey, 2008). the lack of a hierarchical advantage for orientation could be due to the fact that for this dimension two features cannot be processed without mutual interference and this interference interferes with the part-whole processing.

\section{General Discussion}

The present study investigated under which circumstances hierarchical structural information can assist with withindimension conjunction searches. In the first two experiments, participants searched for the conjunction of two topologies. In the part-whole condition, one topology surrounded the other. In the part-part condition, the two topologies were presented side-by-side with no hierarchical relationship. It was found that part-whole topological $\times$ topological conjunction searches were more efficient than part-part topological $\times$ topological conjunction searches. Experiment 3 investigated whether a similar result holds for aspect ratio and found that they did not. Part-whole aspect ratio $\times$ aspect ratio conjunction searches were clearly not efficient, although it was unclear whether or not they were any more efficient than part-part aspect ratio $\times$ aspect ratio conjunction searches. Experiment 4 tested for a part-whole advantage in the orientation domain. Its novel contribution was to directly compare part-whole orientation $\times$ orientation search to part-part orientation $\times$ orientation search, as this had not previously been done. In this way, we were able to confirm not only that part-whole orientation $x$ orientation searches are inefficient but, more importantly, their hierarchical structure affords them no measurable advantage relative to the equivalent part-part orientation $\times$ orientation searches. In fact, we found some evidence that part-whole orientation $\times$ orientation conjunction searches might even be more difficult to perform than part-part orientation $\times$ orientation conjunction searches.

The finding that hierarchical information assists in the within-dimension conjunction search of two colours, two sizes, and two shapes, but not two orientations suggests that the perceptual processing of orientation could be fundamentally different from the processing of colour, size, and shape. Corroborating evidence can be found in studies of visual short-term memory (VSTM) that show that two colours can be registered simultaneously without mutual interference, but two orientations cannot (Becker et al., 2013; Mance et al., 2012). Based on this finding, we hypothesized that for any feature where two examples of that feature cannot be processed simultaneously without mutual interference (e.g. orientation), any within-dimension conjunction searches involving that feature will necessarily be inefficient, regardless of any hierarchical structure. Because two orientations cannot be registered simultaneously (Becker et al., 2013), this would explain why part-whole orientation $\times$ orientation conjunction searches were inefficient. 
A corollary prediction is that if for any feature a hierarchical advantage is observed for within-dimension conjunction searches for that feature (i.e., part-whole searches are found to be more efficient than part-part searches), this would suggest that it should be possible to simultaneously register two examples of that feature without mutual interference. Experiments 5 and 6 tested this prediction in the domains of topology and size. Consistent with this prediction, we found that the topological status of two objects and the sizes of two objects could indeed be registered simultaneously without mutual interference.

It is important to emphasize that the simultaneoussequential paradigm does not measure the processing mechanism directly; it only measures the processing capacity. Therefore, inferences regarding processing architecture must be treated with caution (Townsend, 2004). Although one can use this paradigm to determine whether or not two attributes can be processed without mutual interference, one cannot then determine whether the underlying process is serial or parallel.

Because performance was equivalent in the simultaneous and sequential conditions for colour, topology, and size, it can be inferred that the perceptual system is not capacity-limited in processing these three dimensions, at least for the processing of up to two items simultaneously. Conversely, when performance is better in the sequential condition compared with the simultaneous condition, as was the case for orientation (Becker et al., 2013), this suggests that processing of one item interferes with the processing of another item, such that only a limited amount of information can be processed per unit of time. While it could be the case that the two items must be processed serially, one after another, it also could be the case that observers are able to process the two items simultaneously in parallel, but the items compete for a limited amount of attentional resource. For example, Bilsky and Wolfe (1995) suggested that if two different orientations occupy the same location they will tend to interfere with each other, even when one corresponds to a part and the other a whole. However, contrary to this suggestion, in the orientation $\times$ orientation experiments described by Bilsky and Wolfe (1995), it was always very efficient to search for either a part of a particular orientation or a whole of a particular orientation, even though in both cases a conflicting orientation was always presented at the same location. Therefore, the issue of what causes the mutual interference (i.e. reduction in capacity) when processing orientation information remains unresolved.

To gain a better understanding of why orientation $\times$ orientation conjunction search is different from other withindimension conjunction searches, one would need to employ methods that measure the processing mechanism independent of the capacity. One example of such a method would be Systems Factorial Technology (Townsend \& Nozawa, 1995). Ongoing investigations in our lab are currently using this technique in an attempt to determine whether the processing mechanism for orientation is fundamentally different from that for colour, size, and topology or whether the differences in processing are solely due to capacity differences.

\section{Appendix}

Table 1 Mean error rates for each condition of three experiments

\begin{tabular}{lllll}
\hline & Experiment 1 & Experiment 2 & Experiment 3 & Experiment 4 \\
\hline Part-whole & $2.76 \%$ & $4.81 \%$ & $7.01 \%$ & $16.80 \%$ \\
Part-part & $3.85 \%$ & $4.23 \%$ & $8.47 \%$ & $8.21 \%$ \\
Part-feature & $1.67 \%$ & $1.99 \%$ & $2.08 \%$ & $2.12 \%$ \\
Whole-feature & $1.47 \%$ & $2.12 \%$ & $1.18 \%$ & $1.60 \%$ \\
\hline
\end{tabular}

\section{References}

Becker, M. W., Miller, J. R., \& Liu, T. (2013). A severe capacity limit in the consolidation of orientation information into visual short-term memory. Attention, Perception, \& Psychophysics, 75, 415-425.

Bilsky, A. B., \& Wolfe, J. M. (1995). Part-whole information is useful in visual search for size $\times$ size but not orientation $\times$ orientation conjunctions. Perception and Psychophysics, 57, 749-760.

Brainard, D. H. (1997). The psychophysics toolbox. Spatial Vision, 10, 433-436.

Chen, L. (2005). The topological approach to perceptual organization. Visual Cognition, 12, 553-637.

Chun, M. M., \& Wolfe, J. M. (1996). Just say no: How are visual searches terminated when there is no target present? Cognitive Psychology, 30, 39-78.

Cousineau, D. (2005). Confidence intervals in within-subject designs: A simpler solution to Loftus and Masson's method. Tutorials in Quantitative Methods for Psychology, 1(1), 42-45.

Donnelly, N., Humphreys, G. W., \& Riddoch, M. J. (1991). Parallel computation of primitive shape descriptions. Journal of Experimental Psychology: Human Perception and Performance, 17, 561-570.

Mance, I., Becker, M. W., \& Liu, T. (2012). Parallel consolidation of simple features into visual short-term memory. Journal of Experimental Psychology: Human Perception and Performance, $38,429-438$

Morey, R. D. (2008). Confidence intervals from normalized data: A correction to Cousineau (2005). Tutorial in Quantitative Methods for Psychology, 4(2), 61-64.

Pelli, D. G. (1997). The VideoToolbox software for visual psychophysics: transforming numbers into movies. Spatial Vision, 10, 437-442.

Townsend, J. T. (2004). The serial-parallel dilemma: A case study in a linkage of theory and method. Psychonomic Bulletin \& Review, 11, 391-418.

Townsend, J. T., \& Nozawa, G. (1995). On the spatio-temporal properties of elementary perception: An investigation of parallel, serial, and coactive theories. Journal of Mathematical Psychology, 39, 321-359.

Treisman, A., \& Gelade, G. (1980). A feature-integration theory of attention. Cognitive Psychology, 12, 97-136.

Treisman, A., \& Gormican, S. (1988). Feature analysis in early vision: Evidence from search asymmetries. Psychological Review, 95, 1548. 
Treisman, A., \& Sato, S. (1990). Conjunction search revisited. Journal of Experimental Psychology: Human Perception and Performance, 16, 459-478.

Wolfe, J. M. (1998). What can 1 million trials tell us about visual search? Psychological Science, 9, 33-39.

Wolfe, J. M. (2007). Guided search 4.0: Current progress with a model of visual search. In W. Gray (Ed.), Integrated models of cognitive systems (pp. 99-119). New York: Oxford.

Wolfe, J. M., Cave, K. R., \& Franzel, S. L. (1989). Guided search: An alternative to the feature integration model for visual search. Journal of Experimental Psychology: Human Perception and Performance, 15, 419-433.
Wolfe, J. M., Friedman-Hill, S. R., \& Bilsky, A. B. (1994). Parallel processing of part-whole information in visual search tasks. Perception \& Psychophysics, 55, 537-550.

Wolfe, J. M., \& Horowitz, T. S. (2004). What attributes guide the deployment of visual attention and how do they do it? Nature Reviews Neuroscience, 5, 495-501.

Wolfe, J. M., Yee, A., \& Friedman-Hill, S. R. (1992). Curvature is a basic feature for visual search tasks. Perception, 21, 465-480.

Wolfe, J. M., Yu, K. P., Stewart, M. I., Shorter, A. D., Friedman-Hill, S. R., \& Cave, K. R. (1990). Limitations on the parallel guidance of visual search: Color $\times$ color and orientation $\times$ orientation conjunctions. Journal of Experimental Psychology: Human Perception and Performance, 16, 879-892. 